\title{
Constitutive EGFR Signaling in Oligodendrocyte Progenitors Leads to Diffuse Hyperplasia in Postnatal White Matter
}

\author{
Sanja Ivkovic, ${ }^{1,2}$ Peter Canoll, ${ }^{1}$ and James E. Goldman ${ }^{1,2}$ \\ ${ }^{1}$ Department of Pathology and the ${ }^{2}$ Center for Neurobiology and Behavior, Columbia University, New York, New York 10032
}

\begin{abstract}
Gliogenesis requires the careful orchestration of migration, differentiation, and proliferation of progenitors. Signaling through the epidermal growth factor receptor (EGFR) has been implicated in regulating these processes in a variety of cell types, including neural progenitors. By retroviral infection, we constitutively expressed an EGFR-GFP fusion protein in white matter glial progenitors at postnatal day 3 of the rat forebrain in vivo and analyzed the development of these cells over the subsequent 15 weeks. EGFR-GFP+ cells remained proliferative and migratory, gradually populating the brains ipsilateral and contralateral to the side of viral infection, but never differentiated into mature glia. The accumulation of these cells doubled the total cell density in white matter and led to a 10-fold increase in the abundance of glial progenitors, giving rise to a progenitor "hyperplasia." The marker profile of infected cells, NG2+, olig2+, PDGFR- $\alpha+$, nestin + , GFAP-, and CC1 -, indicated a close resemblance to oligodendrocyte progenitors. Positive immunostaining for phosphorylated EGFR colocalized with punctate accumulation of EGFR-GFP, indicating that a subset of receptors was engaged in active signaling. Furthermore, EGF was required to observe phospho-tyrosine EGFR immunostaining of glial progenitors in culture. These observations suggest that constitutive EGFR expression can inhibit glial differentiation, but requires ligand as well.
\end{abstract}

Key words: epidermal growth factor receptor; glial progenitors; oligodendrocyte progenitors; white matter; glial differentiation; glial migration; glioma

\section{Introduction}

The majority of gliogenesis in the rodent forebrain occurs during the first 2 weeks of postnatal development. During this time, glial progenitors migrate widely through the white matter and cortex, where they differentiate into oligodendrocytes and astrocytes. The molecular mechanisms that regulate glial progenitor cell migration are not well understood, but must be closely linked to their differentiation and proliferation, to assure the appropriate number and distribution of glial cells in the adult nervous system.

Several studies have implicated epidermal growth factor receptor (EGFR) signaling in the migration, proliferation, and differentiation of neural progenitor cells. During embryonic development, EGFR plays a major role in cell migration (Bayer et al., 1991; Burrows et al., 1997, 2000; Caric et al., 2001; Boockvar et al., 2003; Ciccolini et al., 2005), proliferation (Gritti et al., 1999), and differentiation (Lillien, 1995; Viti et al., 2003; Sun et al., 2005), although the effects of EGFR signaling vary with the cell type and developmental time. EGFR-mediated signaling is involved in both radial and tangential modes of migration (Bayer et al., 1991). Moreover, recent studies have shown that the asymmetric distribution of EGFR on the cell membrane influences the final

Received May 7, 2007; revised Dec. 5, 2007; accepted Dec. 12, 2007.

This work was supported by National Institutes of Health Grants NS17125 (J.E.G.), K08-NS045070 (P.C.), and MH157174-28 and NS07062-28 (S.I.). We thank Dr. Sally Temple for the CLE-GFP and EGFR-GFP construct, Drs. Tom Jessell and Bill Stallcup for antibodies, and Drs. Phyllis Faust and Carol Mason for sharing their microscope systems with us.

Correspondence should be addressed to Dr. Sanja Ivkovic, Department of Pathology, Columbia University, 630 West 168th Street, New York, NY 10032. E-mail: si2117@columbia.edu.

DOI:10.1523/JNEUROSCI.4327-07.2008

Copyright $\odot 2008$ Society for Neuroscience $\quad$ 0270-6474/08/280914-09\$15.00/0 cell fate choice of embryonic progenitors (Sun et al., 2005). Additionally, neural progenitors from the adult forebrain subventricular zone (SVZ) also respond to EGFR activation with enhanced proliferation and migration (Craig et al., 1996; Doetsch et al., 2002). Although EGFR has been implicated in the regulation of all of the above-mentioned processes, its precise role in postnatal gliogenesis has not been well established.

Postnatally, EGFR is widely expressed by migrating progenitors, but as cells advance along their differentiation pathways, EGFR expression attenuates (Fox and Kornblum, 2005). Therefore, we hypothesized that a loss of EGFR expression might be required for glial differentiation and thus that extended EGFR expression in glial progenitors might inhibit their differentiation and promote extended migration and proliferation. To test this hypothesis, we infected glial progenitors in the neonatal rat white matter (predominantly oligodendrocyte progenitors) with a retrovirus that expresses an EGFR-GFP fusion protein (Sun et al., 2005), which enabled us to observe the infected cells and the membranous distribution of the receptor.

The EGFR-GFP-expressing progenitors remained highly migratory over an extended period of time (at least 15 weeks), allowing them to reach distant brain structures, mostly by migrating through white matter tracts, which they populated with increasing abundance. EGFR expression also kept them in an immature and proliferative state that resembled oligodendrocyte progenitors, characterized by the expression of NG2, olig2, PDGFR- $\alpha$, and nestin. Over time, the animals developed a diffuse hypercellularity (or hyperplasia) of the white matter, due entirely to the accumulation of these progenitors. In rare instances, we 
A

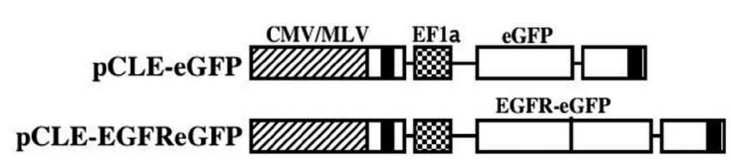

C

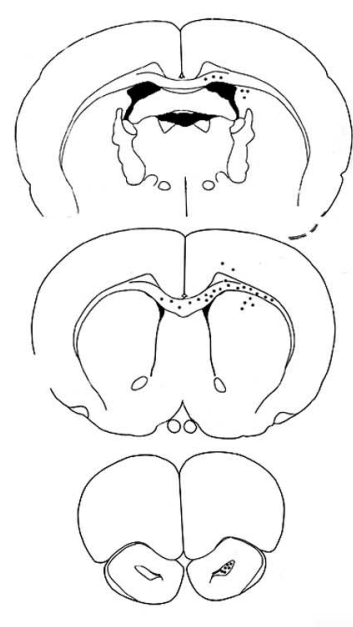

CLE-GFP

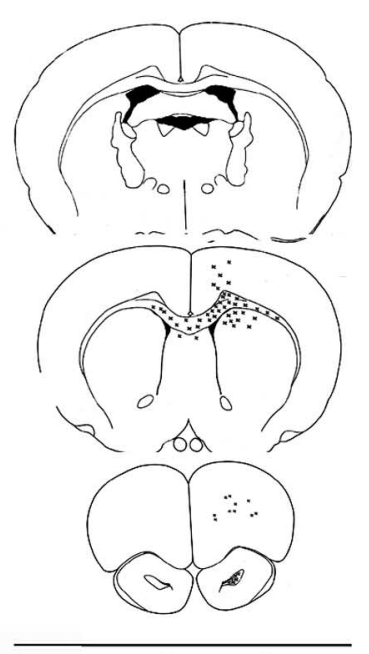

EGFR-GFP
B

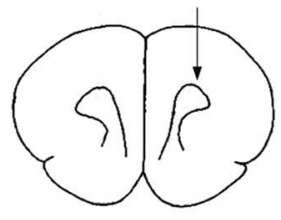

coronal

D

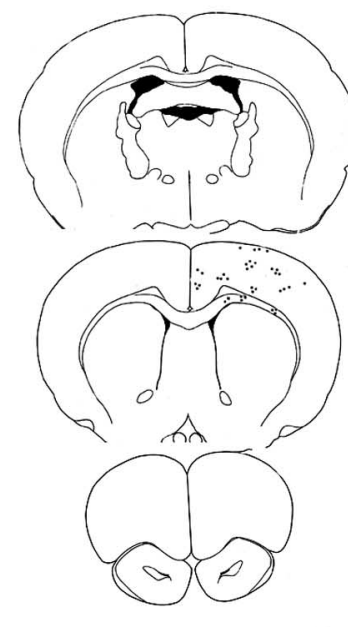

CLE

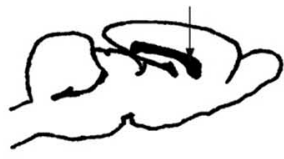

sagittal

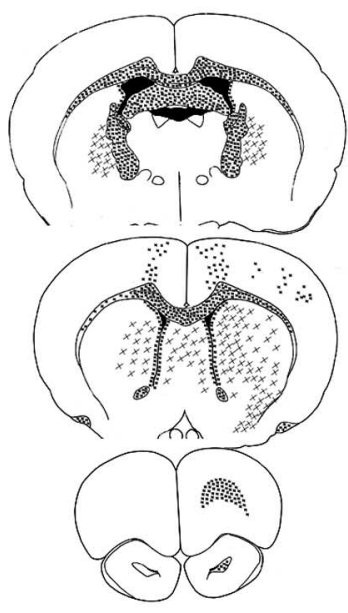

EGFR-GFP

5 DPI

\section{WPI}

E

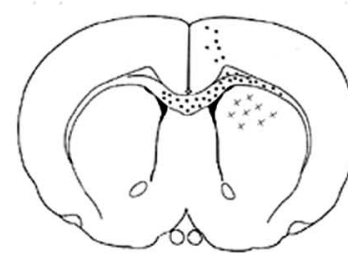

10 DPI

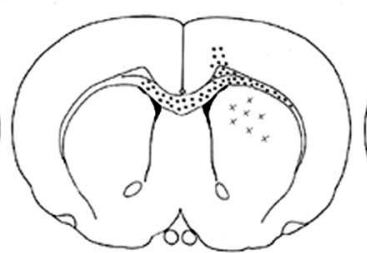

3WPI

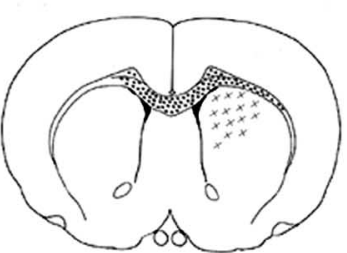

9WPI

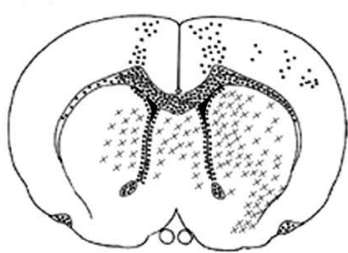

15WPI

EGFR-GFP

Figure 1. EGFR-GFP constitutive expression enhances migration of infected cells. $\boldsymbol{A}, \boldsymbol{B}$, White matter glial progenitor cells were infected with either the control virus pCLE-eGFP or the pCLE-EGFReGFP $(\boldsymbol{A})$ by unilateral viral injection to the rostral white matter $(\boldsymbol{B})$ at P3. C, At 5 dpi, EGFR-GFP + cells had migrated farther than control, CLE-GFP-infected cells, both rostrally and contralaterally through corpus callosum (middle panels). At 15 wpi, CLE-GFP cells have migrated through white matter and cortex, mainly on the ipsilateral side of the injection. $\boldsymbol{D}$, In contrast, EGFR-GFP + cells widely infiltrated the brain both ipsilaterally and contralaterally, mostly along white matter tracts (corpus callosum, fimbria-fornix), reaching rostral white matter and olfactory bulb (bottom right). EGFR-GFP + cells invaded gray matter, but preferentially the ventral structures: caudate-putamen and striatum. $E$, Analysis of the EGFR-GFP + cell distribution at different time points (10 dpi, 3 wpi, 9 wpi, and 15 wpi) showed the continuous increase in the distance that cells had migrated from the injection site.

observed the spontaneous formation of tumor masses composed of EGFR-GFP + cells.

\section{Materials and Methods}

Animal injections. Three-day-old Sprague Dawley rats were anesthetized by immersion in an ice bath for $8 \mathrm{~min}$. Animals' heads were placed in a stereotactic apparatus (Stoelting, Avondale, IL). The bregma was identified, and a burr hole was made $2 \mathrm{~mm}$ lateral and $1 \mathrm{~mm}$ rostral. A 26 gauge Hamilton (Reno, NV) microsyringe was inserted to a depth of $1.9 \mathrm{~mm}$, and $1 \mu \mathrm{l}$ of virus was injected at a rate of $0.2 \mu \mathrm{l} / \mathrm{min}$. Incisions were closed and animals allowed to recover. Brains were analyzed at 5 and $10 \mathrm{~d}$ postinjection (dpi) and 3, 9, and 15 weeks postinjection (wpi). Animals were anesthetized with ketamine-xylazine before cardiac perfusion with PBS and 4\% paraformaldehyde, postfixed for $24-48 \mathrm{~h}$, and then trans- ferred to PBS until use. All animal experiments were performed according to the guidelines of the Institutional Animal Care and Use Committee, Columbia University.

Retrovirus production. EGFR-GFP and CLE-GFP plasmids were generated by Sun et al. (2005) from the original pCLE retroviral vector (Gaiano et al., 1999) and were a kind gift from Dr. Sally Temple (University at Albany, State University of New York, Albany, NY). Replication-deficient viruses with vsv-G coats were generated from these constructs as described previously (Kakita and Goldman, 1999). Viral titers were determined in colony-forming units (CFUs) by incubating C6 glioma cells with serial dilutions of retrovirus in 10-fold steps. At $48 \mathrm{~h}$ postinfection, the number of GFP + cell clusters was counted. The CFUs were calculated by multiplying the number of GFP + cell clusters by the dilution factor. The titer of both the CLE- 


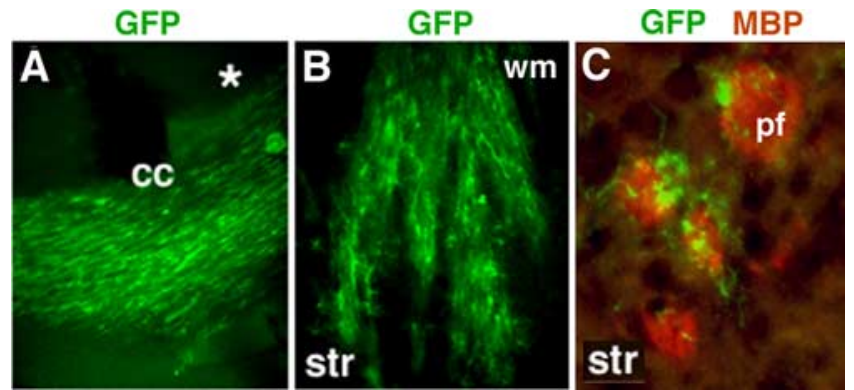

Figure 2. EGFR-GFP + cells migrate preferentially along axonal tracts. $\boldsymbol{A}$, EGFR-GFP + cells migrate along axonal tracts in corpus callosum when crossing to the contralateral side (asterisk indicates side of the retroviral injection). When invading the striatum, EGFR-GFP + cells were following the route of pencil fibers $(\boldsymbol{B})$ and were observed in the close vicinity of the pencil fibers in the striatum (coronal section) (C). wm, White matter; cc, corpus callosum; str, striatum; pf, pencil fibers.

GFP and EGFR-GFP viruses was $10^{6} / \mu \mathrm{l}$. Viruses were injected as described above.

Cell culture. Forebrain subcortical white matter was dissected from Sprague Dawley rats [postnatal day 3 (P3)-P5], dissociated with a $0.025 \%$ trypsin-EDTA/0.25 mg/ml DNase solution, and filtered through a $35 \mu \mathrm{m}$ Nitex mesh. The isolated cells were cultured in B104 conditioned media, as described previously (Canoll et al., 1996), for $2 \mathrm{~d}$, during which time the cultures were infected with the EGFR-GFP retrovirus. B104 medium was then removed, and the cells were replated at 6000 cells/well in an eight-well culture slide (Nunc, Naperville, IL) and grown for either $4 \mathrm{~d}$ in basal medium (DMEM, N2, T3, 0.5\% FBS, and penicillin/streptomycin/amphotericin) or basal medium with EGF (1 ng/ml) (Sigma, St. Louis, MO) for immunostaining with phospho-EGFR antibody or for $1 \mathrm{~d}$ for Ki67 staining. Cultures were fixed in 4\% paraformaldehyde, washed with $1 \times \mathrm{PBS}$, and stored at $4^{\circ} \mathrm{C}$.

Staining procedures. Immunocytochemistry was performed on brain sections from different time points after injections and on cells from the glial preps. Similar results were seen with cell preps from P3 or P5 animals. Fixed brains were cryopreserved in 30\% sucrose in $1 \times$ PBS and cryosectioned at $12 \mu \mathrm{m}$, and sections were stored at $-80^{\circ} \mathrm{C}$ until used. Antibodies used were guinea pig anti-Olig2 (1:100; kind gift from Dr. Thomas Jessel, Columbia University, New York, NY), mouse anti-Nestin (rat 401) (1:100; Developmental Studies Hybridoma Bank, University of Iowa, Iowa City, IA), rabbit anti-GFAP (1:500; DAKO, Carpinteria, CA), rabbit anti-GFP (1:500; Invitrogen, Carlsbad, CA), rabbit anti-Ki67 (1: 1000; NovoCastra, New Castle, UK), rabbit anti-NG2 (1:500; kind gift from Dr. William Stallcup, Burnham Institute for Medical Research, La Jolla, CA), sheep anti-EGFR (1:100; Millipore, Billerica, MA), mouse anti-CC1 (1:50; Merck Biosciences, Darmstadt, Germany), mouse antimyelin basic protein (MBP) (1:1000; Covance, Harrogate, UK), mouse anti-phospho-EGFR-Y1068 (1:50; Cell Signaling Technology, Beverly, MA), rabbit anti-phospho-EGFR-Y1173 (1:100; Stressgen, San Diego, CA), goat anti-PDGFR- $\alpha$ (1:80; R \& D Systems, Minneapolis, MN), mouse anti-O1 (1:100; Developmental Studies Hybridoma Bank), and mouse anti-O4 (1:50; Developmental Studies Hybridoma Bank). Block with $10 \%$ horse serum, $0.1 \%$ Triton $\mathrm{X}-100$ in $1 \times$ PBS was performed for $30 \mathrm{~min}$ before incubation overnight with primary antibody at $4^{\circ} \mathrm{C}$. The following day, the sections were washed extensively and incubated in secondary antibody for $1 \mathrm{~h}$ at room temperature. FITC- and TRITC(Jackson ImmunoResearch, West Grove, PA; Alexa, Invitrogen) conjugated secondary antibodies were used for double fluorescence. Sections were washed extensively again and stained with Hoechst 33342 (Invitrogen), for nuclear visualization.

Microscopy. Histological sections and cell cultures were examined and photographed using a Zeiss Axiophot 200 fluorescence microscope equipped with an Axiocam (Zeiss, Thornwood, NY) and Openlab imaging software (Improvision, Lexington, MA). Micrographs were further processed into composite figures using Adobe (San Jose, CA) Photoshop.

Cell counting. Coronal cryosections $(12 \mu \mathrm{m})$ of brains were examined at the level of the injection site at 15 wpi with the pEGFR-GFP or pCLE. Cells positive for Hoescht nuclear dye were counted in the following way: four $40 \times$ fields from three brains were photographed, and the number of cells staining positive for each marker was manually counted using Adobe Photoshop. Statistical analysis was performed using InStat v3.0 program. Cells grown in culture were counted in a similar manner: ten $20 \times$ fields from two separate experiments were photographed, and the number of cells positive for Ki 67 and GFP was manually counted. The number of double-positive cells was expressed as a percentage of the total number of GFP+ cells per field.

\section{Results}

To analyze the effects of EGFR signaling in vivo, we stereotactically injected either the CLE-GFP retrovirus, as a control, or the EGFR-GFP retrovirus $(1 \mu \mathrm{l})$ (Fig. $1 A)$ into the forebrain subcortical white matter of 3-d-old rats (P3) (Fig. $1 B$ ). Animals were killed at different time points, and brains were processed for immunohistochemical staining on either vibratome $(100 \mu \mathrm{m})$ or cryostat sections $(12 \mu \mathrm{m})$. Cells infected with either control or EGFR-containing virus were bright green under the fluorescence microscope and were easy to identify. The shortest time point analyzed was $5 \mathrm{dpi}$, and the longest was 15 wpi. We also analyzed several time points in between (10 dpi, 3 wpi, and 9 wpi), which allowed us to follow the dispersion of EGFR-GFP cells over time.

\section{EGFR-expressing progenitor cells are highly migratory}

At 5 dpi with the control, CLE-GFP virus, the large majority of GFP+ cells were seen in the subcortical white matter near the injection site. Some cells had migrated short distances from the injection site along the white matter, into the overlying cortex and the striatum. Very few cells crossed the corpus callosum to the contralateral side, consistent with previous results (Kakita et al., 2003). Only rare GFP+ cells were seen in the SVZ and rostral migratory stream (RMS), suggesting that some small amount of retrovirus had diffused into the SVZ underlying the injection site or a few infected cells had migrated from white matter into the SVZ. In contrast, by 5 dpi in the brains infected with the EGFRGFP retrovirus, the GFP + cells had migrated further in the white matter than in the control brains (Fig. 1C). Many more of GFP+ cells had invaded the corpus callosum and had crossed to the other side into the contralateral white matter.

After 15 weeks, the differences were more marked. EGFRGFP + cells were widely dispersed throughout both the ipsilateral and contralateral sides (Fig. 1D). EGFR-GFP+ cells had populated the fornix and internal capsule, migrating both rostrally and caudally. The density of EGFR-GFP+ cells was the highest at the coronal plane of the injection site, gradually tapering off both rostrally and caudally. Considerable numbers of EGFR-GFP+ cells had migrated rostrally along the intrabulbar anterior commissure and into the ipsilateral olfactory bulb. No EGFR-GFP+ cells were found in the RMS. EGFR-GFP + cells were found in the gray matter as well, including neocortex and striatum and septum, largely in the coronal plane of the injection. They populated these same structures on the contralateral side, although to a lesser extent. In the more caudal regions, almost no EGFR-GFP+ cells were found in the cortex, and their distribution in the ventral structures was restricted to the caudate-putamen (Fig. 1D). Comparing brains at different times after injections allowed us to monitor the extent of migration over time. Analysis of the locations of EGFR-GFP + cells at $10 \mathrm{dpi}, 3$ wpi, and 9 wpi showed a progression of EGFR-GFP + cells throughout the white matter (Fig. 1E). 

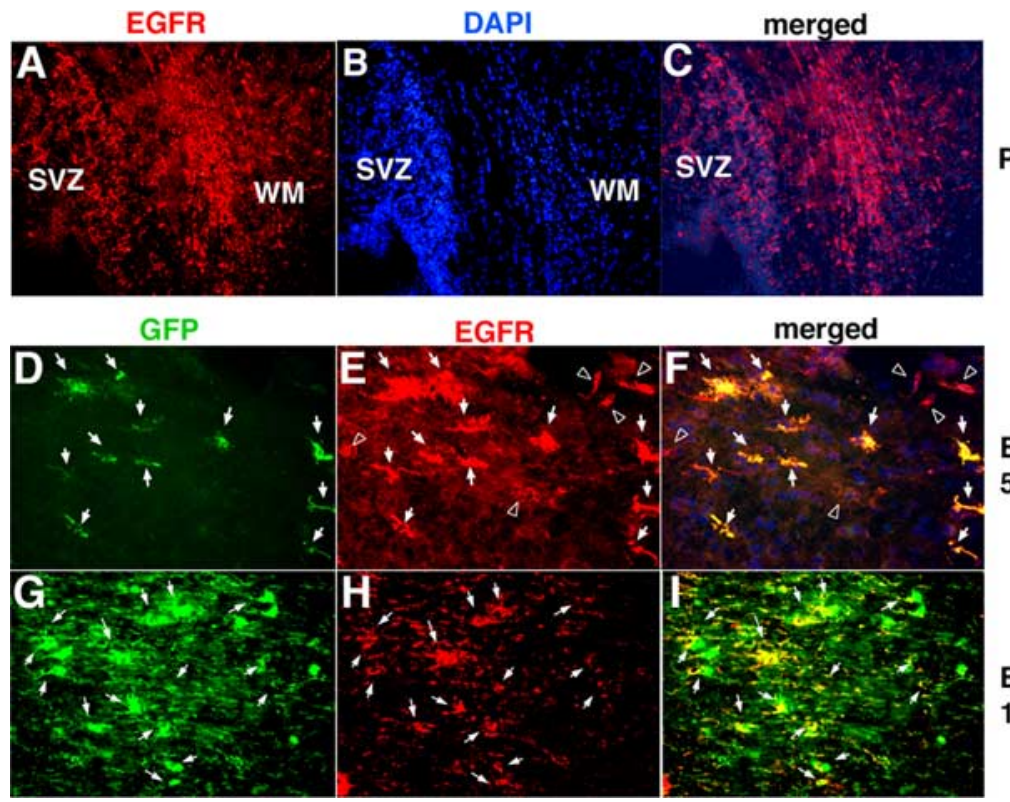

Figure 3. EGFR expression is present in many cells in white matter at the time of injection and is prolonged in the EGFR-GFPinfected cells. $\boldsymbol{A}$, EGFR is expressed in SVZ and white matter (WM) at P3. B, DAPI (Hoescht) staining of the same region as in $\boldsymbol{A}$. $\boldsymbol{C}$, DAPI and EGFR staining merged. D-F, All cells infected with EGFR-GFP were EGFR + at 5 dpi (arrows), but not all EGFR + cells were GFP + (arrowheads). G-I, At 15 wpi, all EGFR-GFP-infected cells were EGFR + . There were no EGFR +/GFP - cells, indicating that endogenous EGFR expression had decreased below detectable levels by that time.

\section{Patterns of migration}

EGFR-GFP + cells did not appear to infiltrate through the brain in a random pattern. These cells preferentially migrated through white matter tracts. EGFR-GFP + cells that crossed the contralateral side either remained in white matter or entered the dorsal cortex in a pattern corresponding to the transcallosal fiber system, axons of which enter the dorsomedial cingulate and motor cortices (Fig. $2 \mathrm{~A}$ ), suggesting that they used axonal tracts to travel as previously reported for glial progenitors (Kakita et al., 2003). Similarly, EGFR-GFP + cells descended into the striatum from subcortical white matter along axonal tracts (pencil fibers) (Fig. $2 B$ ). To highlight this anatomic connection, we performed double labeling for MBP and GFP, which showed the close association between the striatal pencil fibers and EGFR-GFP+ cells (Fig. $2 C)$.

\section{Endogenous EGFR expression in the infected cells}

Retroviral infection of progenitors led to the constitutive expression of EGFR-GFP. To determine whether those cells expressed endogenous EGFR at the time of injection, we performed immunostaining for EGFR in the P3 rat brain. Numerous cells in white matter and in the dorsolateral SVZ were positive for EGFR (Fig. $3 A-C)$. We infected brains with the control, CLE-GFP virus and then stained sections for both GFP and EGFR after $5 \mathrm{~d}$. Approximately $40 \%$ of GFP + cells expressed detectable levels of endogenous EGFR, denoting the presence of EGFR-expressing cells in the population we labeled with retrovirus (data not shown). Double labeling in the EGFR-GFP virus-infected brains at $5 \mathrm{dpi}$ showed strong EGFR labeling in all infected/GFP+ cells (Fig. $3 D-F)$, as expected. EGFR expression was also detected in a subset of uninfected cells (Fig. $3 F$ ). Triple-label immunofluorescence microscopy showed that many of the EGFR +/GFP - cells were uninfected NG2+ glial progenitors (data not shown). At 15 wpi in the EGFR-GFP-infected brains, there was a complete overlap between the GFP and EGFR labeling, signifying that the in-
P3

EGFR-GFP 5 DPI

fected cells continued to express the EGFR-GFP fusion protein. We do not know whether these cells also continued to express endogenous EGFR. However, we were not able to detect EGFR+/GFPcells in white matter (Fig. 3G-I), indicating that endogenous EGFR expression had fallen below detection in uninfected cells. Together, these results suggest that many of infected cells were expressing endogenous EGFR around the time of the infection, that the levels of endogenous EGFR subside during normal gliogenesis, and that the EGFR-GFP retrovirus produced a continued expression of the viral-derived EGFR throughout the entire 15 week interval. The long-term expression appears to be robust, because we find EGFR-GFP expression even 1 year after infection (data not shown and supplemental Fig. 2, available at www.jneurosci.org as supplemental material).

\section{EGFR overexpression in vivo inhibits} the final differentiation of progenitors To analyze the effects of EGFR overexpression on the fates of infected cells, we stained cryosections obtained from animals infected with EGFR-GFP or CLE-GFP retroviruses and killed at 5 dpi and 15 wpi for cell lineage markers. In white matter, we examined the expression of nestin, olig2, vimentin, GFAP, CC1, PDGFR- $\alpha$, Ki67, and NG2. At 5 dpi, EGFR-GFP-infected cells showed characteristics of immature glia: they were all positive for olig2 (Fig. $4 \mathrm{~B}$ ), which is expressed in neonatal forebrain glial progenitors (Marshall et al., 2005). The EGFRoverexpressing cells were also nestin + (Fig. $4 \mathrm{~A}$ ), in contrast to the CLE-GFP+ cells, which did not express nestin (data not shown). Although a few of the CLE-GFP-infected cells were GFAP positive (data not shown), the majority of the control and EGFR-expressing cells were GFAP- (Fig. $4 F$ ), which indicates that these cells, at least at this early time, had not reached the stage of astrocyte development when they express GFAP (Zerlin et al., 2004). Almost all of the EGFR-GFP+ cells were NG2 + (Fig. 4C) and PDGFR- $\alpha+$ (Fig. $4 D$ ), consistent with an early stage in the oligodendrocyte lineage (Hall et al., 1996; Nishiyama, 2001). None of the EGFR-GFP+ cells was $\mathrm{CC} 1+$, indicating that they had not reached the stage of a more mature oligodendrocyte (Fig. $4 E$ ). Immunostaining for Ki67 showed that both EGFR-GFPand CLE-GFP-infected cells were proliferative (40\% and 20\%, respectively, measured in the area of the subcortical white matter, located dorsal to the striatum) (Fig. 4G, data not shown).

At 15 wpi, all of the EGFR-GFP + cells expressed markers characteristic for oligodendrocyte progenitors; they were all olig2,$+ \mathrm{NG} 2+$, and PDGFR- $\alpha+$ (Fig. $5 B-D$ ). In addition, some of the cells were nestin + (Fig. $5 A$ ). None of the cells morphologically resembled myelinating oligodendrocytes or astrocytes (Fig. 5). None expressed CC1 or GFAP (Fig. $5 E, F$ ). The EGFR-GFP+ cells displayed long, lacy, sometimes elaborate processes (Fig. 5) and populated the white matter in far larger numbers than the control cells (Fig. 1). In contrast, by 15 wpi, most of the control, CLE-GFP virus-infected cells in white matter had differentiated into myelinating oligodendrocytes, displaying the characteristic myelin sheath labeling (supplemental Fig. 1, available at www. 
jneurosci.org as supplemental material). Those cells that had migrated into the cortex and striatum had developed either into astrocytes or oligodendrocytes with radially oriented, lacy process patterns, but none of them exhibited the features of immature and/or undifferentiated/progenitor cells. Immunohistochemical analysis of CLE-GFP + cells in white matter showed that these cells were nestin-, mostly $\mathrm{NG} 2-, \mathrm{CC} 1+$, and GFAP - [supplemental Fig. 1 (available at www.jneurosci.org as supplemental material) and data not shown]. Few of these cells expressed olig2, which is present in a subpopulation of oligodendrocytes in the mature brain (data not shown). CLE-GFP + cells at 15 wpi displayed a differentiated morphology and were positive for CC1, a marker for mature oligodendrocytes. In contrast to the control virus-infected cells, which did not show Ki67 labeling, we found at 15 wpi that EGFR-GFP + cells were still proliferative. The Ki67 labeling index was highly variable; we found the highest labeling index ( $\sim 30 \%$ of the total number of GFP + cells) in the white matter dorsal to the striatum (Fig. 5G).

EGFR expression results in a progenitor cell hyperplasia in white matter

At all times there appeared to be an increase in total cell density in white matter, as measured by DAPI nuclear staining. To quantitate this phenomenon, we counted total cell numbers and GFP+ cell numbers in the midpoint of the callosum at the level of the injection site at 15 wpi (Fig. $6 A-D$ ). The total cell count in this area of white matter of rats infected with the EGFR-GFP virus was more than twice that in controls (4970 \pm 182 per $\mathrm{mm}^{2}$ vs $2176 \pm 64$ per $\left.\mathrm{mm}^{2}\right)$, and the excess cell number was made up entirely of GFP + cells. The Ki67 labeling data in vivo and in vitro (see below) suggests that the increased progenitor numbers are in part a result of continued, increased proliferation.

\section{Exogenous EGFR-GFP is actively}

signaling in vivo and in vitro

We looked at 15 wpi for phosphorylated EGFR, by immunostaining, as an indication of active signaling. Phospho-EGFR staining in vivo was located on the cell processes, overlapping with the areas of increased GFP density, indicating that at least some of the receptors were phosphorylated and active, or closely associated with activated endogenous receptor (Fig. 7A$C)$. Puncta of GFP accumulations suggest that the receptor density is increased in these regions, possibly because of receptor

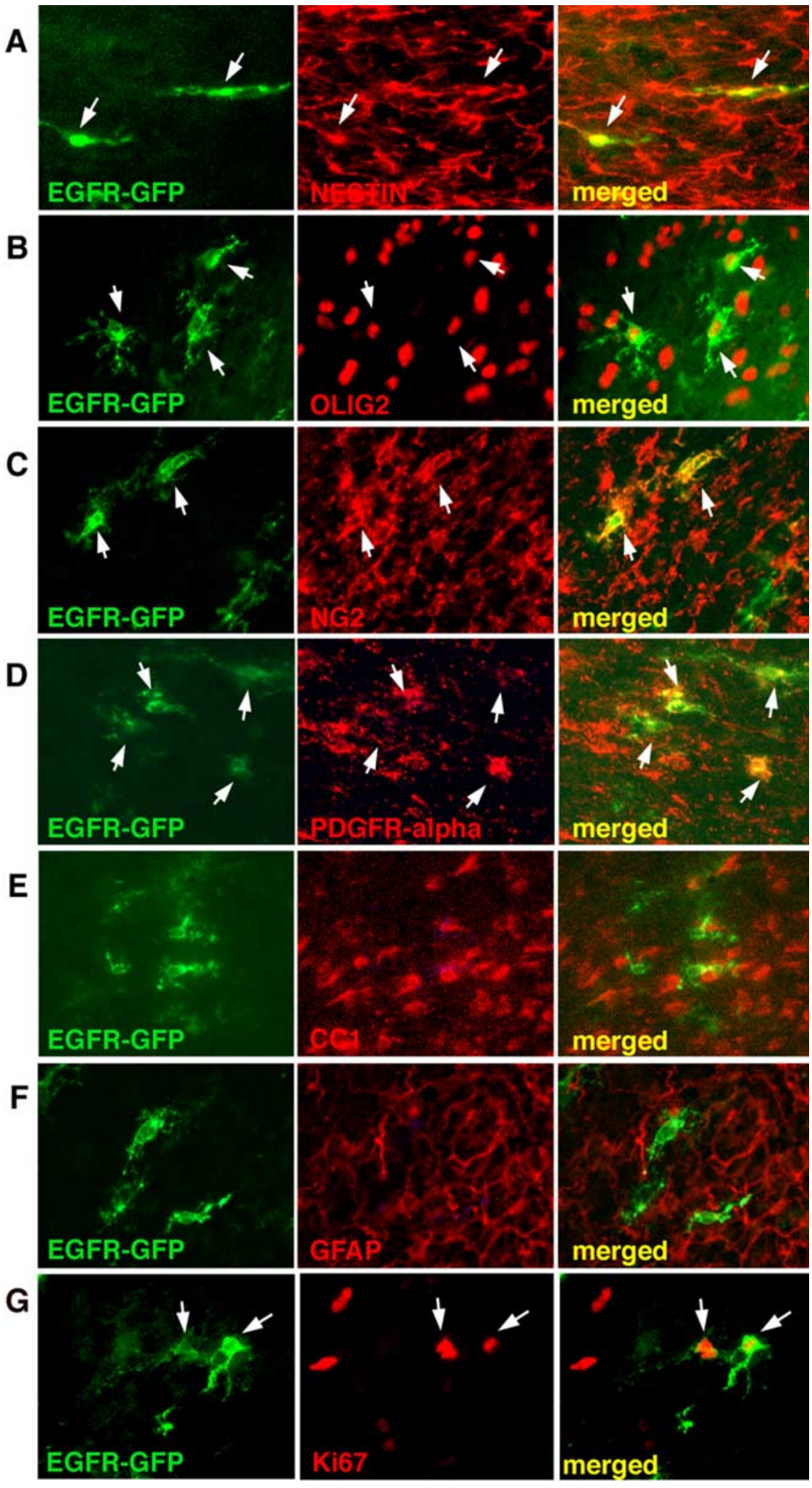

Figure 4. EGFR-GFP + cells express markers of immature glial progenitors at 5 dpi. EGFR-GFP + cells express markers for progenitors (nestin; $\boldsymbol{A}$ ) and glial progenitors (olig2; $\boldsymbol{B}$ ). EGFR-GFP + cells are positive for markers of early oligodendrocytes [NG2 $(\boldsymbol{C})$ and PDGFR- $\alpha(\boldsymbol{D})]$ and negative for markers of oligodendrocytes $(\mathrm{CC} ; \boldsymbol{E})$ and mature astrocytes (GFAP; $\boldsymbol{F})$. Some EGFR-GFP + cells were proliferative (Ki67+; G). Arrows indicate double-labeled cells.

clustering, which leads to receptor activation (Bazley and Gullick, 2005) (Fig. 7A). These findings also indicate that only a small fraction of EGFR-GFP was phosphorylated, at least on this particular tyrosine residue (Y1068). Because tyrosine phosphoryla- 
A
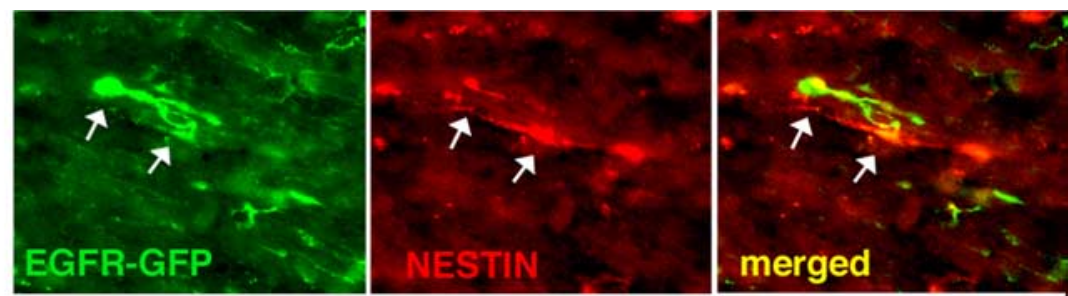

B
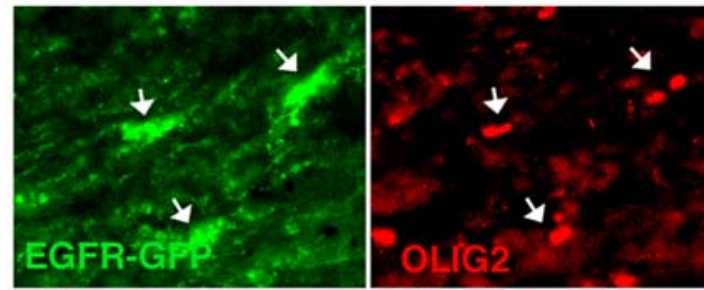

C

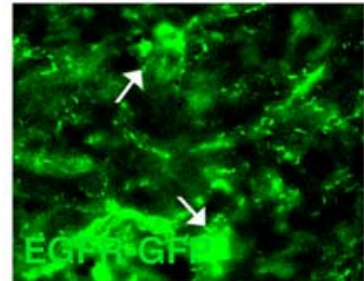

D
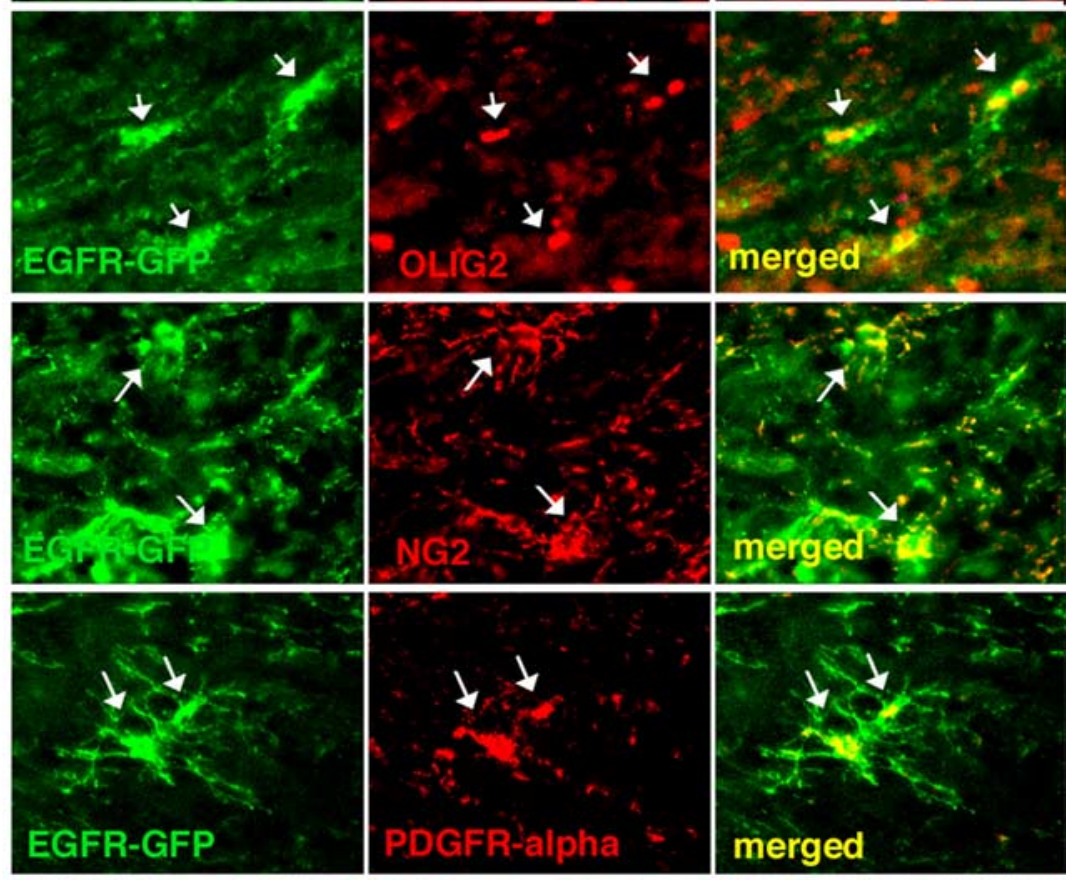

$\mathbf{E}$
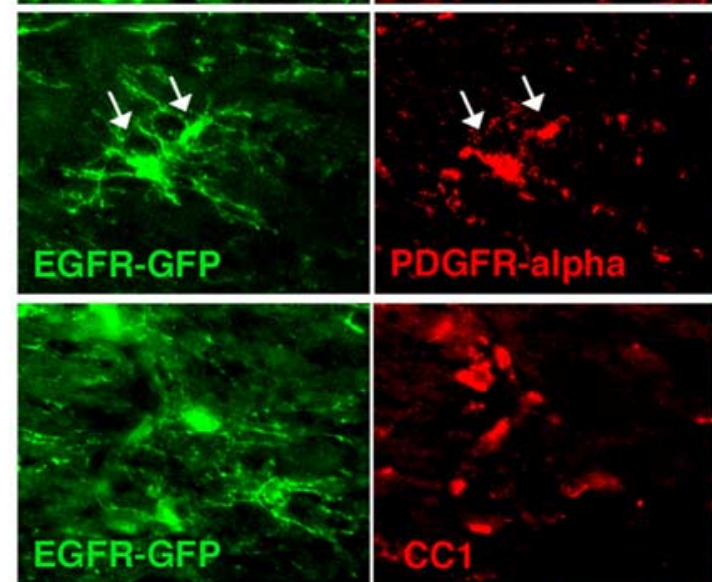

$\mathbf{F}$
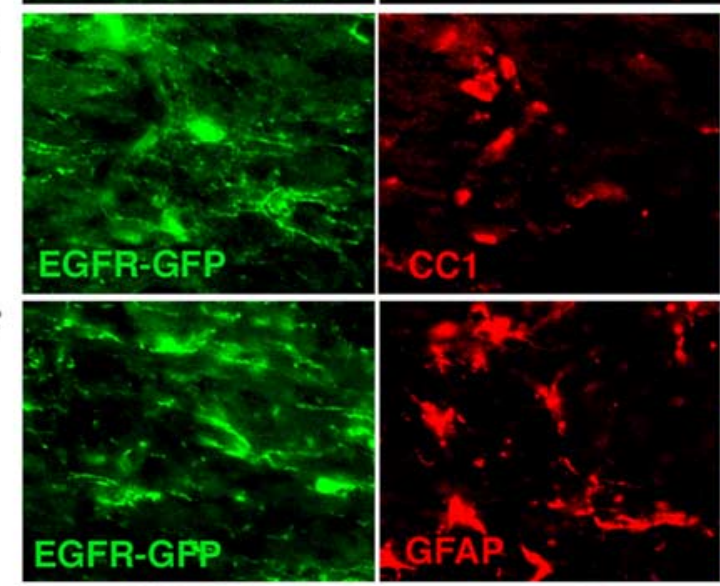

G
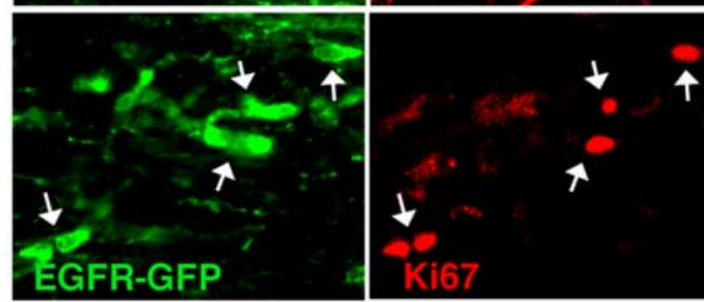
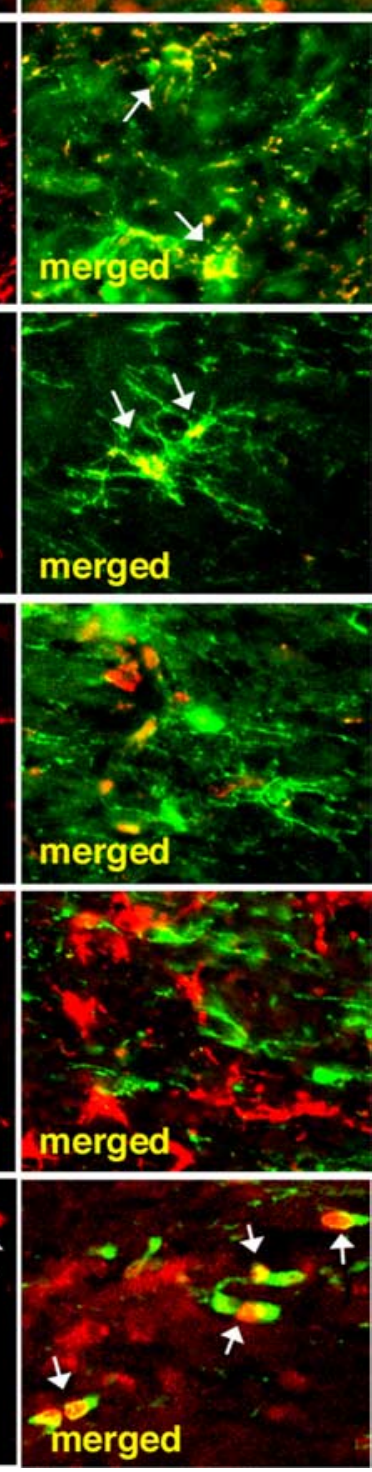

Figure 5. EGFR-GFP + cells resemble immature glial progenitors at 15 wpi. $\boldsymbol{A}-\boldsymbol{D}$, They still expressed general markers for progenitors (nestin; $\boldsymbol{A}$ ) and glial progenitors (olig2; $\boldsymbol{B}$ ) and markers for early oligodendrocytes [NG2 (C) and PDGFR- $\alpha(\boldsymbol{D})] . \boldsymbol{E}, \boldsymbol{F}$, None of the EGFR-GFP + cells expressed markers for either mature oligodendrocytes (CC1; $\boldsymbol{E})$ or mature astrocytes (GFAP; $\boldsymbol{F}) . \boldsymbol{G}$, Some of EGFR-GFP + cells were still proliferative at 15 wpi and Ki67 +. Arrows indicate double-labeled cells.

tion is a reversible event, this may reflect dynamic changes in receptor clustering and activation.

As another way to test whether the EGFR-GFP is actively signaling in infected cells, we isolated progenitors from P5 white

matter, infected them with the EGFR-GFP retrovirus in vitro, and then grew them in basal medium with or without EGF ( $1 \mathrm{ng} /$ $\mathrm{ml}$ ) for the next $4 \mathrm{~d}$. EGFR-GFP+ cells grown in the presence of EGF demonstrated a strong increase in phosphorylation of EGFR, shown through immunostaining for two phospho-tyrosines (Y1068 and Y1173), which colocalized with puncta of GFP expression (Fig. 7G$L$ ), thus confirming that EGFR-GFP is actively signaling as a response to the ligand. Furthermore, double immunostaining for the phospho-Y1173 and phospho-Y1068 residues showed that both tyrosines were phosphorylated in the presence of EGF and were both colocalized with the puncta of GFP (Fig. 7J-L). There was an overlap between the foci of GFP accumulation and the immunostaining for both of the EGFR phospho-tyrosine (Fig. 7J). In contrast, there was little or no phospho-EGFR staining in cells grown in the absence of EGF (Fig. 7D-F).

\section{EGFR-GFP signaling affects glial progenitor differentiation and proliferation in vitro}

We further analyzed the effects of EGFR signaling on the proliferation and differentiation of progenitors in vitro. Glial progenitors were infected in vitro with the EGFR-GFP virus as noted above, and proliferation was assayed after $4 \mathrm{~d}$ with or without EGF. Those grown in the presence of EGF displayed a higher Ki67 labeling index than those grown without EGF $(33.3 \pm 1.62 \%$ with EGF for $4 \mathrm{~d} ; 14.72 \pm$ $1.68 \%$ without EGF). This result is consistent with our findings in vivo (see above) and suggests again that increased proliferation is in part responsible for the progenitor hyperplasia. We also examined the differentiation of oligodendrocytes in these cultures. EGFR-GFP-expressing cells were analyzed for the presence of the $\mathrm{O} 4$ and $\mathrm{O} 1$ oligodendrocyte markers. In the presence of EGF, a smaller proportion of infected cells acquired the $\mathrm{O} 4$ and $\mathrm{O} 1$ antigens (14\% O4 + with EGF vs 40\% O4+ without EGF; $18 \%$ O1+ with EGF vs $41 \%$ O1+ without EGF). Therefore, the presence of the ligand appears to have a pronounced effect on the differentiation of the EGFRGFP-expressing progenitors in vitro.

\section{Discussion}

Constitutive EGFR expression drives migration of progenitor cells

EGFR signaling regulates migration in a variety of cell types, including neuronal cells, fibroblasts, pancreatic epithelial cells, and Drosophila oocytes (Blay and Brown, 1985; Chen et al., 1994; Li et al., 1999, 2000; Miettinen et al., 2000; Caric et al., 


\section{corpus callosum at $15 \mathrm{wpi}$}
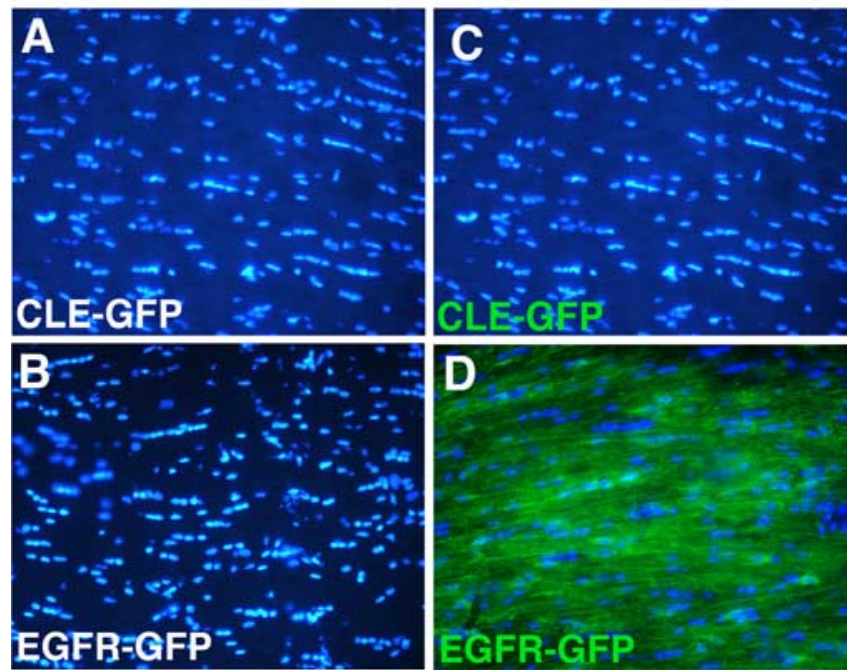

Figure 6. Increased cell number in corpus callosum in EGFR-GFP-infected animals at 15 wpi is a consequence of the increase in the number of GFP + cells only. Cell nuclei were stained with Hoescht dye (blue) $(\boldsymbol{A}-\boldsymbol{D})$, and GFP + cells fluoresce green $(\boldsymbol{C}, \boldsymbol{D})$. At 15 wpi in CLE-GFP-infected animals $(\boldsymbol{C})$, there were no GFP + cells in the center of the corpus callosum. In contrast, in EGFR-GFP-infected animals GFP + cells had widely infiltrated the callosum in large numbers (D). The total cell number in the corpus callosum was more than twice that of control, and the excess cell number was made up entirely of GFP + cells (see Results).

2001; Duchek and Rorth, 2001). In the EGFR-null mice, CNS progenitors accumulate in proliferative zones (Threadgill et al., 1995), suggesting that EGFR signaling may be required for migration during development.

Constitutive EGFR-GFP expression in postnatal progenitors kept them highly migratory, allowing them to infiltrate the brain to distances far from the site of retroviral infection. The distribution of labeled cells was not random, but rather occurred predominantly along white matter tracts. Many EGFR-GFP+ cells had migrated across the corpus callosum into the contralateral hemisphere. In addition, we observed some labeled cells in the ipsilateral cortex, likely to have ascended on radial glia during the first 2 weeks of postnatal life (Zerlin et al., 2004), similar to the control-infected cells. Glial progenitors infected with control virus migrate along similar routes, but to a much more limited extent (as seen in Kakita et al., 2003). Thus, EGFR expression does not appear to alter the directional or substrate preferences of migrating progenitors, but keeps the cells migratory for an extended period of time, allowing them to migrate greater distances than control cells. The prolonged migratory capacity of EGFR$\mathrm{GFP}+$ cells may be a result of the increased density of the receptor on the membrane, allowing the cell to sense whatever minute amounts of the ligand exist in the surrounding areas (Caric et al., 2001).

\section{Constitutive EGFR expression inhibits differentiation of glial progenitors}

Glial progenitors with constitutive EGFR expression failed to differentiate. Based solely on the expression of markers, the EGFR$\mathrm{GFP}+$ cells were immature, exhibiting the closest resemblance to precursors of oligodendrocyte lineage (NG2+, olig2 +, PDGFR$\alpha+$, GFAP-, and CC1 - ). EGFR-GFP + cells were also nestin + . Glial progenitors in vitro and in vivo will upregulate nestin expression in response to growth factor stimulation (Gallo and Armstrong, 1995; Canoll et al., 1999; Assanah et al., 2006).
Whereas CLE-GFP+ cells have already exited the cell cycle and have differentiated at $15 \mathrm{wpi}, \sim 30 \%$ of the total EGFR-GFP+ cells were $\mathrm{Ki} 67+$, although the proliferation index varied from region to region. We do not yet understand these variations, but they may reflect nonhomogeneous ligand(s) distribution in the brain.

EGFR expression is regulated during brain development. After an initial upregulation in germinal zones [ventricular zone (VZ) and SVZ] at midgestation, EGFR expression starts to decline after birth and into adulthood (Seroogy et al., 1995; Fox and Kornblum, 2005). Constitutive expression of EGFR in the embryonic VZ results in early overrepresentation of astrocytes, higher proliferation rates, and enhanced migration (Burrows et al., 1997). Similarly, it forces embryonic day 18 retinal progenitors to differentiate preferentially into Muller glia (Lillien and Wancio, 1998). Surprisingly, we found that abnormally prolonged EGFR expression in postnatal white matter progenitors does not cause the infected cells to differentiate into astrocytes. Furthermore, infected cells exhibited an arrest in their differentiation at a stage resembling oligodendrocyte progenitors. Thus, EGFR stimulation appears to have different effects on embryonic versus postnatal progenitors. This may reflect the differences in the types of progenitors infected, the progenitors' intrinsic capacity to respond to EGFR stimulation, or differences in the environmental context. For example, other signaling molecules, such as FGF and BMP (which are known to affect glial differentiation), can regulate the way progenitor cells respond to EGFR stimulation (Lillien and Raphael, 2000). Studying the effects of these growth factors separately and in combination should provide important insights into how different glial progenitors integrate these signals during normal brain development and how imbalances in these signals can lead to abnormalities in glial migration, proliferation, and differentiation.

Continuous EGFR signaling in white matter glial progenitors leads to hyperplasia and requires ligand

Our results show that overexpression of EGFR can give rise to a diffuse hyperplasia of the white matter composed of cells that closely resemble immature oligodendrocyte progenitors. In most cases, these lesions cause relatively subtle histological changes characterized by an increase in cell density without grossly distorting the brain architecture. The extent of these hyperplastic lesions would be difficult to determine if not for the GFP fluorescence allowing for definitive identification of infected cells.

We observed phosphorylation of the receptor in vivo in a proportion of EGFR-GFP + cells, which strongly suggests that there are sufficient amounts of the ligand(s) in vivo to activate EGFR. Similarly, in cell cultures obtained from early postnatal white matter, the presence of EGF resulted in the phosphorylation of EGFR-GFP (Fig. 7), confirming that EGFR-GFP is actively signaling as a response to the ligand. As a result, EGFR-GFP-infected cells grown in the presence of the ligand showed a decrease in their differentiation and an increase in proliferation when compared with the EGFR-GFP+ cells grown without the ligand. All together, these observations suggest that the effects of EGFR expression in progenitors depend on the presence of an endogenous ligand or ligands. The nature and levels of such ligands in the adult CNS remain to be determined.

Several attempts to stimulate EGFR signaling in vivo have resulted in different outcomes, probably because of different technical approaches and different receptor types. For example, overexpression of the constitutively active receptor mutant, EGFR VIII, in specifically targeted nestin + or GFAP + cells using a TVA 

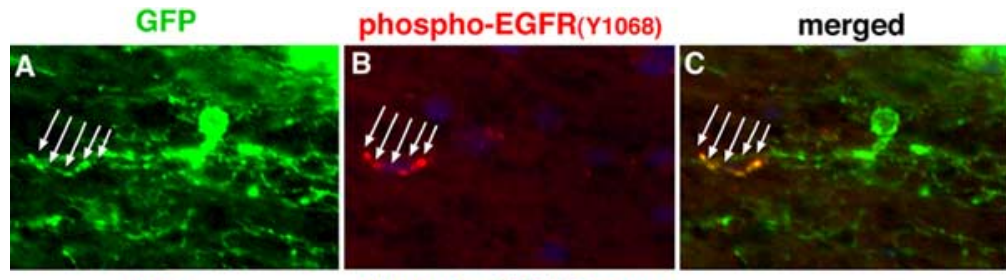

EGFR-GFP

15 WPI
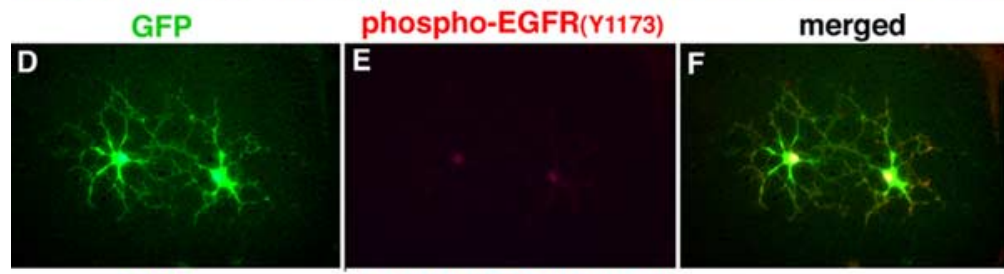

EGFR-GFP

P5

4DIV

w/o EGF

GFP
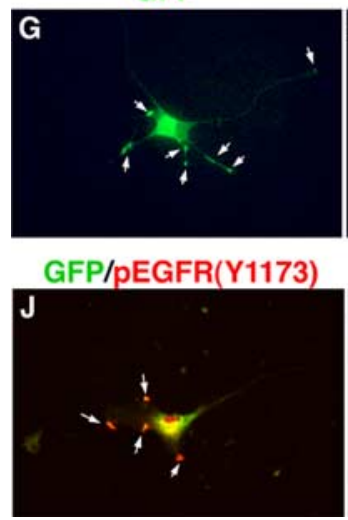

phospho-EGFR(Y1173)
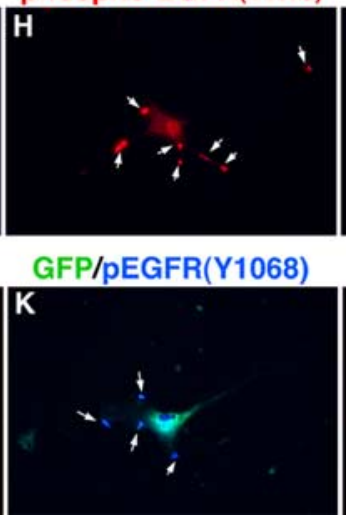

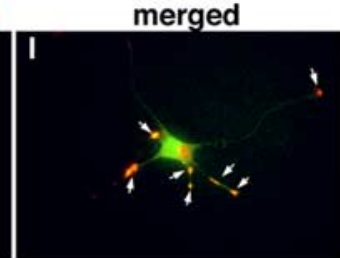

merged

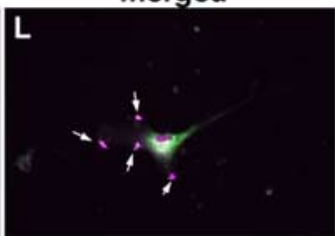

2007). Furthermore, they found that EGFR overexpression caused a reduction in the number of astrocytes and an increase in the number of oligodendrocytes seen in the demyelinating lesion, suggesting that EGFR overexpression may inhibit astrocyte differentiation and induce progenitors to differentiate along the oligodendrocyte lineage. We did not find any astrocytes in vivo, but we cannot conclude that the EGFR expression inhibited astrocyte differentiation, because the very large majority of cells initially infected belonged to the oligodendrocyte and not the astrocyte lineage. Indeed, when we analyzed progenitor cells infected with the EGFR-GFP virus in culture, we did find green, GFAP + cells, presumably originating from proliferating astrocyte precursors in the cultures, but found the same proportions of these cells with and without EGF (our unpublished observations).

The deregulation of EGFR signaling is often manifested in human gliomas ( $\mathrm{Col}-$ lins, 1995; Rao and James, 2004), and previous animal studies have shown that the expression of mutant EGFR VIII can cooperate with other genetic lesions to facilitate the formation of gliomas (Holland et al., 1998; Wei et al., 2006). However, the functional role of EGFR signaling in glioma formation is not understood. In this study, we show that the constitutive expression of EGFR-GFP in glial progenitors is sufficient to keep glial progenitors immature, proliferative, and migratory for an extended time and that this results in the consistent formation of diffuse hypercellular lesions that resemble the diffusely in-

EGFR-GFP-infected cells signal actively in vivo an in vitro. $A-C$, GFP was found in specific foci on the EGFR-GFP-infected cells in white matter $(\boldsymbol{A})$, consistent with receptor accumulation. $\boldsymbol{B}, \boldsymbol{C}$, Some of the GFP + accumulations were also positive for anti-phospho-EGFR (Y-1068) antibody. D-I, Glial progenitors infected with EGFR-GFP and grown in the basa media for $4 \mathrm{~d}$ showed basal levels of EGFR phosphorylation (Y1173; $\mathbf{D}-\boldsymbol{F})$, but in the presence of EGF $(1 \mathrm{ng} / \mathrm{ml} ; \mathbf{G}-\boldsymbol{I})$, the level of phosphorylation increased dramatically. GFP signal on the membrane overlaps with the positive immunostaining for P-EGFR (arrows). J-L, Double immunostaining for two phosphorylated tyrosine residues (Y1068 and Y1173) (arrows).

retroviral system, appeared not to generate any kind of hyperplastic cell population (Holland et al., 1998). The choice of the receptor mutant, EGFR VIII, which does not bind the ligand, in combination with the restricted target cell population may have led to the different outcome than in our study. In a different approach, NG2 + cells were modified to overexpress hEGFR either using transient transfection or using a transgenic mouse in which EGFR is transcribed under the control of the CNPase promoter. The NG2+/hEGFR/GFP+ and NG2+/CNPase-hEGFR/ GFP + cells showed increased migratory potential after being transplanted into the lateral ventricles of postnatal rats, but the cells did not appear to form accumulations or cause any other kind of histological abnormalities Indeed, these cells had differentiated by 4 weeks after transplantation, preferentially into astrocytes, but also into oligodendrocytes, depending on their final destination (Aguirre et al., 2005), an observation that contrasts with our findings that EGFR-expressing cells do not differentiate. However, the amount of EGFR introduced into cells, the extent of its expression after transient transfection versus retroviral infection, the choice of the targeted cell population, and the experimental manipulations of the cells before and during the transplantation may have enabled NG2 + cells to differentiate over time. Despite the differences in approach, these combined observations suggest that constitutive EGFR signaling is sufficient to promote and maintain progenitor migration. More recently, the same group has shown that EGFR overexpression increased the proliferation and migration of NG2+/Olig2+ glial progenitors in response to demyelinating lesions (Aguirre et al., filtrative component seen in human gliomas. Furthermore, some animals formed either multiple, small, proliferative nodules in the white matter (15 wpi and 6 months postinjection) (supplemental Fig. 2A, $B$, available at www.jneurosci.org as supplemental material) or large tumors composed of EGFR-GFP + cells after a long period postinjection (from 6 months to 1 year) (supplemental Fig. 2. $C, D$, available at www.jneurosci.org as supplemental material). Still, the majority of animals never developed tumors, but rather the diffuse hypercellularity. This stands in sharp contrast to the very rapid and consistent formation of malignant tumors that is seen when glial progenitors are infected with retroviruses that express the PDGF- $\beta$ ligand (Assanah et al., 2006). Interestingly, the PDGF-infected cells show a similar immunophenotype (NG2+/Olig2+/PDGFR+/Nestin+/GFAP-). Additional studies are necessary to understand the formation of tumors in EGFR-GFP injected brains. Nevertheless, the continued proliferation of EGFR-GFP + cells and their inability to differentiate may provide a fertile ground for the accumulation of further genetic abnormalities causing tumor formation.

\section{References}

Aguirre A, Rizvi TA, Ratner N, Gallo V (2005) Overexpression of the epidermal growth factor confers migratory properties to nonmigratory postnatal neural progenitors. J Neurosci 25:11092-11106.

Aguirre A, Dupree JL, Mangin JM, Gallo V (2007) A functional role of EGFR signaling in myelination and remyelination. Nat Neurosci 10:990-1002. Assanah M, Lochhead R, Ogden A, Bruce J, Goldman J, Canoll P (2006) 
Glial progenitors in adult white matter are driven to form malignant gliomas by platelet-derived growth factor-expressing retroviruses. J Neurosci 26:6781-6790.

Bayer SA, Altman J, Russo RJ, Dai XF, Simmons JA (1991) Cell migration in the rat embryonic neocortex. J Comp Neurol 307:499-516.

Bazley LA, Gullick WJ (2005) The epidermal growth factor receptor family. Endocr Relat Cancer 12:S17-S27.

Blay J, Brown KD (1985) Epidermal growth factor promotes the chemotactic migration in cultured rat intestinal epithelial cells. J Cell Physiol 124:107-112.

Boockvar JA, Kapitonov D, Kapoor G, Schouten J, Counelis GJ, Bogler O, Snyder EY, McIntosh TK, O’Rourke DM (2003) Constitutive EGFR signaling confers a motile phenotype to neural stem cells. Mol Cell Neurosci 24:1116-1130.

Burrows RC, Wancio D, Levitt P, Lillien L (1997) Response diversity and the timing of progenitor cell maturation are regulated by developmental changes in EGFR expression in the cortex. Neuron 19:251-267.

Burrows RC, Lillien L, Levitt P (2000) Mechanisms of progenitor maturation are conserved in the striatum and cortex. Dev Neurosci 22:7-15.

Canoll P, Kraemer R, Teng KK, Marchionni MA, Salzer JL (1999) GGF/ Neuregulin induces a phenotypic reversion of oligodendrocytes. Mol Cell Neurosci 13:79-94.

Canoll PD, Musacchio JM, Hardy R, Reynolds R, Marchionni MA, Salzer JL (1996) GGF/Neuregulin is a neuronal signal that promotes the proliferation and survival and inhibits the differentiation of oligodendrocyte precursors. Neuron 17:229-243.

Caric D, Raphael H, Viti J, Feathers A, Wancio D, Lillien L (2001) EGFRs mediate chemotactic migration in the developing telencephalon. Development 128:4203-4216.

Chen P, Gupta K, Wells A (1994) Cell movement elicited by epidermal growth factor receptor requires kinase and autophosphorylation but is separable from mitogeneses. J Cell Biol 124:547-555.

Ciccolini F, Mandl C, Holzl-Wenig G, Kehlenbach A, Hellwig A (2005) Prospective isolation of late development multipotent precursors whose migration is promoted by EGFR. Dev Biol 284:112-125.

Collins VP (1995) Gene amplification in human gliomas. Glia 15:289-296.

Craig CG, Tropepe V, Morshead CM, Reynolds BA, Weiss S, van der Kooy D (1996) In vivo growth factor expansion of endogenous subependymal neural precursor cell populations in the adult mouse brain. J Neurosci 16:2649-2658.

Doetsch F, Petreanu L, Caille I, Garcia-Verdugo J-M, Alvarez-Buylla A (2002) EGF converts transit-amplifying neurogenic precursors in the adult brain into multipotent stem cells. Neuron 36:1021-1034.

Duchek P, Rorth P (2001) Guidance of cell migration by EGF receptor signaling during Drosophila oogenesis. Science 291:131-133.

Fox IJ, Kornblum HI (2005) Developmental profile of ErbB receptors in murine central nervous system: implications for functional interactions. J Neurosci Res 79:584-597.

Gaiano N, Kohtz JD, Turnbull DH, Fishell G (1999) A method for rapid gain-of-function studies in the mouse embryonic nervous system. Nature 2:812-819.

Gallo V, Armstrong R (1995) Developmental and growth factor-induced regulation of nestin in oligodendrocyte lineage cells. J Neurosci 15:394-406.

Gritti A, Frolichsthal-Schoeller P, Galli R, Parati EA, Cova L, Pagano SF, Bjornson CR, Vescovi AL (1999) Epidermal and fibroblast growth factors behave as mitogenic regulators for a single multipotent stem cell-like population from the subventricular region of the adult mouse forebrain. J Neurosci 19:3287-3297.

Hall A, Giese NA, Richardson WD (1996) Spinal cord oligodendrocytes develop from ventrally derived progenitor cells that express PDGF alphareceptors. Development 122:4085-4094.

Holland EC, Hively WP, DePinho RA, Varmus HE (1998) A constitutively active epidermal growth factor receptor cooperates with disruption of G1 cell-cycle arrest pathways to induce glioma-like lesions in mice. Genes Dev 12:3675-3685.

Kakita A, Goldman JE (1999) Patterns and dynamics of SVZ cell migration in the postnatal forebrain: monitoring living progenitors in slice preparations. Neuron 23:461-472.

Kakita A, Zerlin M, Takahashi H, Goldman JE (2003) Some glial progenitors in the neonatal subventricular zone migrate through the corpus callosum to the contralateral cerebral hemisphere. J Comp Neurol 458:381-388.

Li J, Lin ML, Wiepz GJ, Guadarrama AG, Bertics PJ (1999) Integrinmediated migration of murine B82L fibroblasts is dependent on the expression of and intact epidermal growth factor receptor. J Biol Chem 274:11209-11219.

Li J, Kim YN, Bertics PJ (2000) Platelet-derived growth factor-stimulated migration of murine fibroblasts is associated with epidermal growth factor receptor expression and tyrosine phosphorylation. J Biol Chem 275:2951-2958.

Lillien L (1995) Changes in retinal cell fate induced by overexpression of EGF receptor. Nature 377:158-162.

Lillien L, Raphael H (2000) BMP and FGF regulate the development of EGFresponsive neural progenitor cells. Development 127:4993-5005.

Lillien L, Wancio D (1998) Changes in epidermal growth factor receptor expression and competence to generate glia regulate timing and choice of differentiation in the retina. Mol Cell Neurosci 10:296-308.

Marshall CA, Novitch BG, Goldman JE (2005) Olig2 directs astrocyte and oligodendrocyte formation in postnatal subventricular zone cells. J Neurosci 25:7289-7298.

Miettinen PJ, Huotari M, Koivisto T, Ustinov J, Palgi J, Rasilainen S, Lehtonen E, Keski-Oja J, Otonkoski T (2000) Impaired migration and delayed differentiation of pancreatic islet cells in mice lacking EGFreceptors. Development 127:2617-2627.

Nishiyama A (2001) NG2 cells in the brain: a novel gill cell population. Hum Cell 14:77-82.

Rao RD, James CD (2004) Altered molecular pathways in gliomas: and overview of clinically relevant issues. Semin Oncol 31:595-604.

Schlessinger J (2000) Cell signaling by receptor tyrosine kinases. Cell 103:211-225.

Seroogy KB, Gall CM, Lee DC, Kornblum HI (1995) Proliferative zones of postnatal brain express epidermal growth factor receptor mRNA. Brain Res 670:157-164.

Sun Y, Goderie SK, Temple S (2005) Asymmetric distribution of EGFR receptor during mitosis generates diverse CNS progenitor cells. Neuron 45:873-886.

Threadgill DW, Dlugosz AA, Hansen LA, Tennenbaum T, Lichti U, Yee D, LaMantia C, Mourton T, Herrup K, Harris RC, Barnard JA, Yuspa SH, Coffey RJ, Magnuson T (1995) Targeted disruption of mouse EGF receptor: effect of genetic background on mutant phenotype. Science 269:230-234

Viti J, Feathers A, Phillips J, Lillien L (2003) Epidermal growth factor receptor control competence to interpret leukemia inhibitory factor as an astrocyte inducer in developing cortex. J Neurosci 23:3385-3393.

Wei O, Clarke L, Scheidenhelm DK, Qian B, Tong A, Sabha N, Karim Z, Bock NA, Reti R, Swoboda R, Purev E, Lavoie JF, Bajenaru ML, Shannon P, Herlyn D, Kaplan D, Henkelman RM, Gutmann DH, Guha A (2006) High-grade glioma formation results from postnatal pten loss or mutant epidermal growth factor receptor expression in a transgenic mouse glioma model. Cancer Res 66:7429-7437.

Zerlin M, Milosevic A, Goldman JE (2004) Glial progenitors of the neonatal subventricular zone differentiate asynchronously, leading to spatial dispersion of glial clones and to the persistence of immature glia in the adult mammalian CNS. Dev Biol 270:200-213. 